\title{
Determination of two acrylates in environmental water by high performance liquid chromatography
}

\author{
Yue Qiu ${ }^{1}$, Qing Zhang ${ }^{1}$, Genrong Li ${ }^{1 *}$, Yingkun Gong ${ }^{1}$ and Mei Long ${ }^{1}$ \\ ${ }^{1}$ Chongqing Academy of Metrology and Quality Inspection, 401123 Chongqing, China.
}

\begin{abstract}
A high performance liquid chromatography (HPLC) method was established for the determination of two acrylate substances in environmental water. The optimal chromatographic conditions were determined via exploring the effects of chromatographic column, mobile phase, column temperature, flow rate, detection wavelength and other factors on the separation effect of acrylate substances. Finally, the effective separation of methyl methacrylate and isopropyl methacrylate was realized within 6 min. The retention time of the target compound was used for qualitative analysis and the external standard method was used for quantitative analysis in the experiment. The linear relationship between the two acrylates was good in the range of $0.2-50.0 \mathrm{mg} / \mathrm{L}$, and the correlation coefficient of standard curve was higher than 0.999 . The recovery rate was $88.6 \%-105.3 \%$, the relative standard deviation was $1.7 \%-4.1 \%$, and the detection limit (LODs) was $0.03-0.05 \mathrm{mg} / \mathrm{L}$. The method was simple, efficient and accurate, and suitable for the determination of acrylates in environmental water samples.
\end{abstract}

\section{Introduction}

Acrylate substances are an important chemical raw material, which mainly used as intermediates in organic synthesis [1-3]. There are many monomers prepared from acrylic polymers, including acrylic monomers, methacrylic monomers and other unsaturated double bond compounds that can participate in the copolymerization of acrylic monomers. Acrylates are toxic to a certain extent and can irritate the human body, eyes, mucosa and respiratory tract after inhalation, oral administration or absorption through the skin $[4,5]$. As acrylates are widely used in the chemical industry, they may pollute the surrounding environment through migration and bring about health risks.

At present, the detection of acrylates mainly focused on the environmental atmosphere, textiles, soil, adhesives and medical treatment, while there were few reports on the detection of residues in environmental water. The main detection methods of acrylate substances include gas chromatography (GC) [6-8] and gas chromatography-mass spectrometry (GC-MS) $[9,10]$.

In this work, two acrylates in environmental water were determined by high performance liquid chromatography (HPLC). This method showed the advantages of simple pretreatment, high stability and good accuracy, which could provide data reference for the future detection of acrylate in environmental water.

\section{Materials and methods}

\subsection{Materials and reagents}

Methyl acrylate and isobutyl methacrylate standards were purchased from Dr. Ehrenstorfer (Augsburg, Germany). HPLC grade acetonitrile was supplied from Merck (Darmstadt, Germany).

\subsection{Instruments and equipment}

The experiment was conducted on Agilent 1260 high performance liquid chromatograph (Agilent, USA), SQP Electronic analytical balance (Sedris, Germany) and Advantage A10 Pure water meter (Miliipore, USA).

\subsection{Preparation of standard solution}

The standard substance of $10.0 \mathrm{mg}$ was accurately weighed to glass test tube of $10 \mathrm{~mL}$, then dissolved and volume-stabilized with methanol to prepare a standard storage solution with concentration of $1000 \mathrm{mg} / \mathrm{L}$. The mixed standard working solutions of $0.2 \mathrm{mg} / \mathrm{L}, 1.0 \mathrm{mg} / \mathrm{L}$, $5.0 \mathrm{mg} / \mathrm{L}, 20.0 \mathrm{mg} / \mathrm{L}$ and $50.0 \mathrm{mg} / \mathrm{L}$ were prepared with ultrapure water by step dilution method for HPLC analysis.

\subsection{Sample collection and preparation}

Water samples from different environments were collected and put into a brown glass sampling bottle with

\footnotetext{
" Corresponding author: ligenrongcq@163.com
} 
sealed plug. The samples were stored at low temperature and transported to the laboratory, and then put into a refrigerator at $4^{\circ} \mathrm{C}$ for refrigeration. The test was completed within two weeks. The collected water samples were shaken well and let stand for $30 \mathrm{~min}$. The supernatant was filtered by a $0.45 \mu \mathrm{m}$ organic membrane filter for liquid chromatography analysis.

\subsection{Chromatographic condition}

An Agilent ZORBAX SB-AQ column (250 mm $\times 4.6 \mathrm{~mm}$, $5 \mu \mathrm{m})$ was used. The mobile phase was water and acetonitrile. The column temperature was $30^{\circ} \mathrm{C}$, the detection wavelength was $210 \mathrm{~nm}$, and the flow rate was $1.0 \mathrm{~mL} / \mathrm{min}$. Gradient elution procedure was $0-10 \mathrm{~min}$, $50 \%-100 \%$ B.

\section{Result and analysis}

\subsection{Selection of chromatographic conditions}

The effects of Agilent Eclipse plus C18, Agilent Eclipse XDB-C18, Agilent Extend C18, and Agilent ZORBAX SB-AQ column on the separation of the two acrylates were investigated. The results showed that the degree of separation and peak shape of the two substances on ZORBAX SB-AQ column were more excellent than other columns.

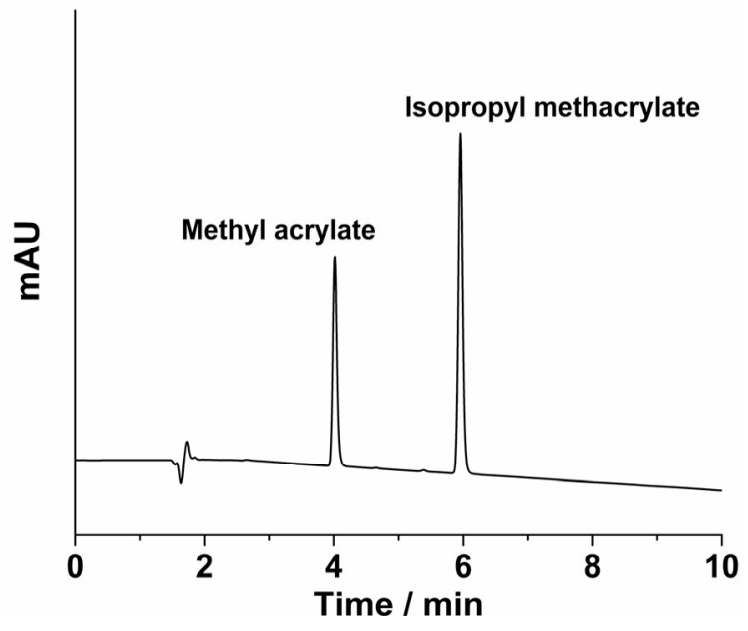

Fig. 1. Chromatogram of standard solution of two acrylate compounds

The effects of the chromatographic conditions of mobile phase, column temperature, flow rate and detection wavelength on the separation of acrylate substances were further explored. The experimental results indicated that the target substances had the best peaking effect in the acetonitrile-water system. The column temperature and flow rate had little influence on the separation degree of the two acrylate substances. The optimal conditions were $30^{\circ} \mathrm{C}$ and $1.0 \mathrm{~mL} / \mathrm{min}$. Both acrylates obtained the maximum UV absorption at 210 $\mathrm{nm}$. As shown in Figure 1, methyl acrylate and isopropyl methacrylate could achieve good baseline separation within 6 min under optimal chromatographic conditions.

\subsection{Standard curve, LOD and LOQ}

Two acrylates of different mass concentrations were mixed with standard working solution and tested under optimal conditions. The linear relationship between the response peak area $(\mathrm{Y})$ and the mass concentration $(\mathrm{X})$ of the target compound was obtained by using the external standard method. The results were listed in Figure 2. Within the linear range of $0.2-50.0 \mathrm{mg} / \mathrm{L}$, the linear correlation coefficients (R2) of methyl acrylate and isopropyl methacrylate were 0.9994 and 0.9999 respectively, indicating a good linear relationship. The detection limits (LODs) and quantitative limits (LOQs) of the two acrylates were severally calculated by using the methods of 3 -fold signal-to-noise ratio $(\mathrm{S} / \mathrm{N} \geqslant 3)$ and 10 -fold signal-to-noise ratio $(\mathrm{S} / \mathrm{N} \geqslant 10)$. As shown in Table 1, the LODs of methyl methacrylate and isopropyl methacrylate were $0.05 \mathrm{mg} / \mathrm{L}$ and $0.03 \mathrm{mg} / \mathrm{L}$, respectively. The LOQs of the two acrylates were both $0.2 \mathrm{mg} / \mathrm{L}$.
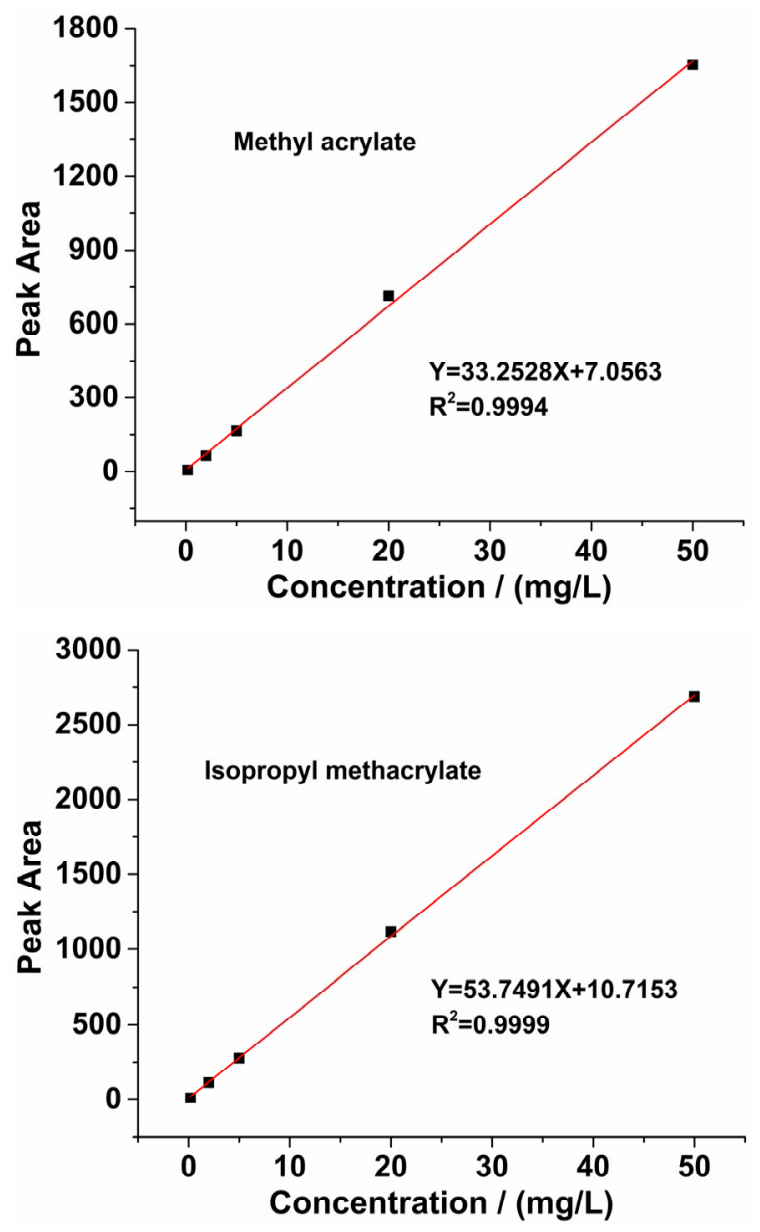

Fig. 2. Standard curves of the standard solution of two acrylates 
Table 1. Linear range, LODs and LOQs of two acrylates.

\begin{tabular}{|c|c|c|c|c|}
\hline No. & Compound & $\begin{array}{c}\text { Linear range } \\
(\mathbf{m g} / \mathbf{L})\end{array}$ & $\begin{array}{c}\text { LOD } \\
(\mathbf{m g} / \mathbf{L})\end{array}$ & $\begin{array}{c}\mathbf{L O Q} \\
(\mathbf{m g} / \mathbf{L})\end{array}$ \\
\hline 1 & methyl acrylate & $0.2-50.0$ & 0.05 & 0.2 \\
\hline 2 & $\begin{array}{c}\text { isopropyl } \\
\text { methacrylate }\end{array}$ & $0.2-50.0$ & 0.03 & 0.2 \\
\hline
\end{tabular}

\subsection{Precision and recovery}

The blank ambient water samples were selected for standard recovery and precision test, and three acrylic esters solution with different mass concentrations $(0.2$, 5.0 and $50.0 \mathrm{mg} / \mathrm{L}$ ) were added to the sample solution. The test was repeated for 6 times at each concentration level. The experimental results were listed in Table 2 . The results showed that the recoveries $(R)$ of the two acrylate compounds at the three standard levels of high, medium and low were in the range of $88.6 \%-105.3 \%$, and the relative standard deviations (RSDs) were between $1.7 \%$ and $4.1 \%$, which could meet the actual detection requirements.

Table 2. Recoveries $(R)$ and RSDs $(n=6)$ for acrylates

\begin{tabular}{|c|c|c|c|c|c|c|}
\hline \multirow{2}{*}{ Com-pound } & \multicolumn{2}{|c|}{$\mathbf{0 . 2} \mathbf{~ m g} / \mathbf{k g}$} & \multicolumn{2}{c|}{$\mathbf{5 . 0} \mathbf{~ m g} / \mathbf{k g}$} & \multicolumn{2}{c|}{$\mathbf{5 0 . 0} \mathbf{~ m g} / \mathbf{k g}$} \\
\cline { 2 - 7 } & $\begin{array}{c}\mathbf{R} \\
/ \%\end{array}$ & $\begin{array}{c}\mathbf{R} \\
\mathbf{S}\end{array}$ & $\begin{array}{c}\mathbf{R} \\
\mathbf{D}\end{array}$ & $\begin{array}{c}\mathbf{R} \\
\mathbf{S}\end{array}$ & $\begin{array}{c}\mathbf{R} \\
\mathbf{D}\end{array}$ & $\begin{array}{c}\mathbf{R} \\
\mathbf{S}\end{array}$ \\
\hline methyl acrylate & 88.6 & 4.1 & 105.3 & 3.4 & 98.9 & 1.7 \\
\hline $\begin{array}{c}\text { isopropyl } \\
\text { methacrylate }\end{array}$ & 92.0 & 3.7 & 96.7 & 2.9 & 102.5 & 2.2 \\
\hline
\end{tabular}

\subsection{Actual sample test}

The selected actual samples included two water samples from different rivers, two industrial wastewater samples and one tap water sample. The experimental results showed that acrylate residues were found in one river water sample and two industrial wastewater samples. The chromatograms of actual samples were shown in Figure 3, and the contents of methyl acrylate and isopropyl methacrylate were in the range of $0.95-3.88$ $\mathrm{mg} / \mathrm{L}$.

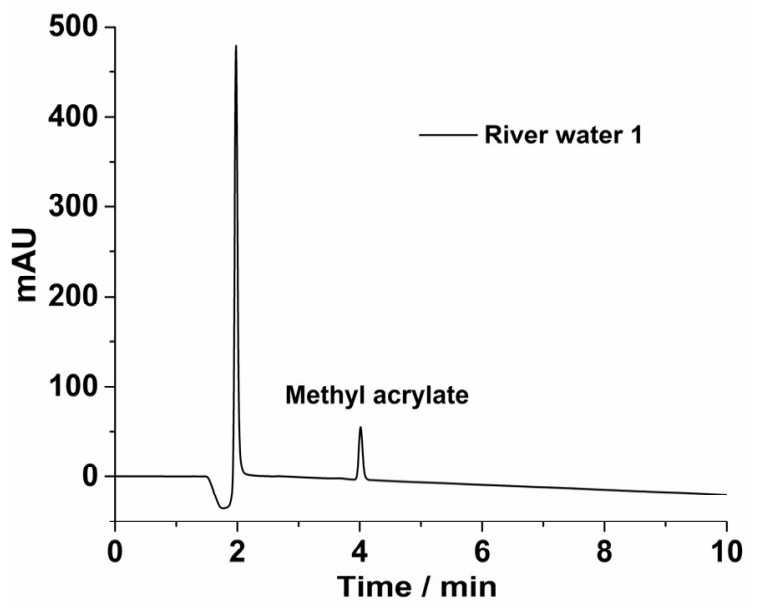

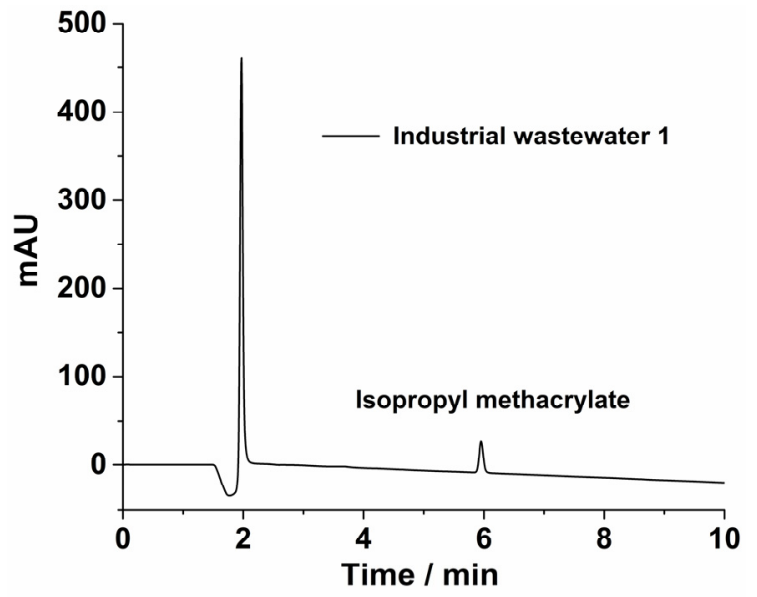

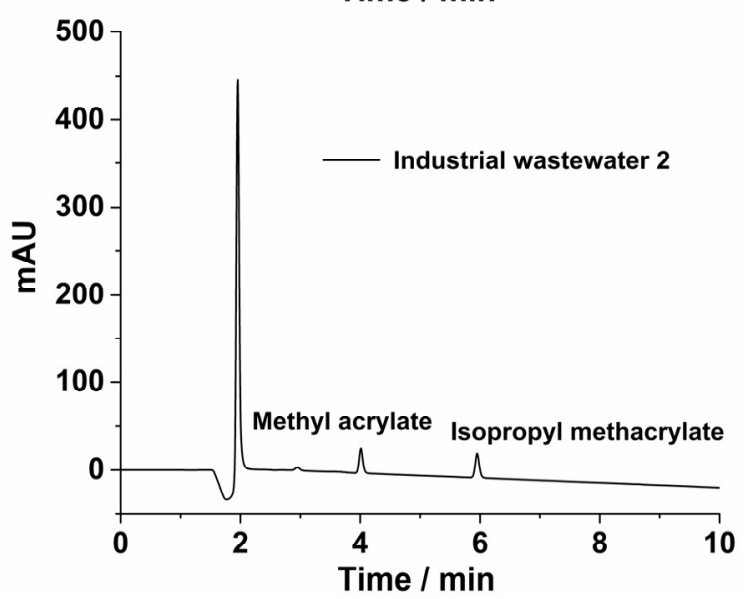

Fig. 3. Chromatogram of two acrylate substances in the actual samples 


\section{Conclusions}

A HPLC method for the determination of two acrylate substances in environmental water was established. This method could separate methyl acrylate and isopropyl methacrylate within $6 \mathrm{~min}$. The linear correlation coefficients of the two target substances were more than 0.999, and the technical indexes such as detection limit, quantitative limit, recovery rate and precision could meet the detection requirements of actual samples. This method had good stability and high accuracy, which can provide a new and reliable method supporting for the risk assessment of organic pollutants in environmental water bodies in the future.

\section{Funding}

This research was funded by Scientific Research Project of Chongqing Market Supervision Administration [CQSJKJ2019005].

\section{References}

1. H.Y. Zhao, G.F. Cao, X.L. Zhang, et al., Phys. Test Chem. Anal. Part B: Chem. Anal. 45, 6 (2009)

2. Z. Wei, H. Huang, Chem. Bioeng. 26, 8 (2009)

3. X.Y. Sengtu, W. Zhang, Dye. Finish. 34, 5 (2008)

4. S.G Gong, B. Kong, S.X. Tuo, et al., Chin. J. Chromatogr. 31, 10 (2013)

5. D. Liu, X.Q. Chen, M.J. Wu, et al., Chin. J. Chromatogr. 29, 12 (2011)

6. H.Z. Zhu, Occup and Health 25, 23 (2009)

7. L.F. Sun, Q.N. Xun, Q. Zhang, et al., Chem. Anal. Meter. 24, 3 (2015)

8. H.Z. Zhu, W.D. Cheng, W.L. Huang, et al., Chinese J. Health Lab. Technol. 5 (2010)

9. D. Liu, X.Q. Chen, M.J. Wu, et al., Phys. Chin. J. Chromatogr. 29, 12 (2011)

10. Y. Lai, R. Lin, L. X. Cai, et al., Chin. J. Chromatogr. 30, 1 (2012) 